Research Article

\title{
The distribution and characteristics of Extended-Spectrum $\beta$-Lactamase (ESBL) producing Escherichia coli and Klebsiella species among urinary isolates in a tertiary care hospital
}

\author{
DMBT Dissanayake ${ }^{1}, \mathrm{SSN}_{\text {Fernando }}{ }^{1}, \mathrm{NS}_{\text {Chandrasiri }}{ }^{2}$ \\ Sri Lanka Journal of Infectious Diseases 2012 Vol.2(2);30-36 \\ DOI: http://dx.doi.org/10.4038/sljid.v2i2.4235 \\ Keywords : ESBL, Urinary tract infections, Escherichia coli, Klebsiella spp.
}

\begin{abstract}
A prospective study was carried out to determine the distribution of ESBL producing uropathogens in different units of a tertiary care hospital in Colombo and in community acquired UTIs presenting to this hospital. A total of 2303 urine samples from adult patients were received in the microbiology laboratory of the hospital during a four-month period, of which 626 were significant positive cultures. Escherichia coli and Klebsiella species accounted for $46 \%(\mathrm{n}=286)$ of the total isolates with the majority $(\mathrm{n}=228,80 \%)$ being $E$. coli. Thirty three percent $(\mathrm{n}=94)$ of these isolates produced extended-spectrum $\beta$-lactamases (ESBLs). Half (50\%) of the Klebsiella isolates were ESBL producers while $29 \%$ of the E. coli produced ESBLs. Ten of 11 isolates in the intensive care unit and 6 of 8 in the genito-urinary unit were ESBL producers. The ESBL rate in the general medical and surgical wards and the out-patient department were 33\% and 14\% respectively. Out of the 181 isolates from community acquired UTIs, 13\% produced ESBLs. Resistance to multiple drugs was considerably higher in ESBL positives compared to ESBL negative isolates. Urinary catheters, diabetes mellitus, previous antibiotic use, urinary tract abnormalities and recurrent urinary tract infections were associated with a higher risk of acquiring ESBL producing organisms $(\mathrm{P}<0.001)$.
\end{abstract}

\section{Introduction}

Urinary tract infections (UTIs) are among the most common conditions causing individuals to seek medical care. Antibiotic treatment for UTI is usually given empirically before the laboratory results of urine culture are available, or sometimes without doing a urine culture in uncomplicated, community acquired infections. Changing epidemiology and ever-increasing antimicrobial resistance in uropathogens can cause difficulties in choice of appropriate empirical treatment.

\footnotetext{
${ }^{1}$ University of Sri Jayewardenepura, Sri Lanka

${ }^{2}$ Colombo South Teaching Hospital, Kalubowila
}

Address for correspondence: Dr. Thushari Dissanayake . Dept of Microbiology, Faculty of Medical Sciences, University of Sri Jayewardenepura, Sri Lanka. Phone: 0772987942 E mail: 
Enterobacteriaceae including Escherichia coli and Klebsiellae are the leading cause of UTI. ${ }^{1}$ Production of extended-spectrum $\beta$-lactamases (ESBLs) has been identified as a major cause of drug resistance in these bacteria. ESBL producing strains are resistant to the penicillins, oxyimino-cephalosporins and monobactams. ${ }^{2}$ Furthermore, plasmid mediated ESBL resistance is commonly associated with co-resistance to many other antibiotics including aminoglycosides, co-trimoxazole and quinolones which are common non $\beta$-lactam antibiotics used for treatment of complicated and uncomplicated UTI.

Once largely a problem in the hospital setting, infections caused by ESBL-producing E. coli in the community have been a major concern worldwide in recent times, UTI being the commonest presentation. ${ }^{3,4,5}$ Detection of ESBL production by urinary isolates is therefore very important to ensure appropriate antibiotic treatment. Up to date information on the antibiotic sensitivity of organisms causing UTI is also mandatory for formulation and updating of guidelines for empirical treatment. As the prevalence of ESBLs differs significantly both geographically and with different risk factors in patients, knowledge of these variations can help in appropriate and timely antibiotic therapy as well as avoidance of unnecessary antibiotic use.

With reports of high prevalence of ESBL production in members of Enterobacteriaceae and paucity of information in Sri Lanka, the present study was undertaken to determine the distribution of ESBL producing uropathogens in different units of a tertiary care hospital in Colombo and in community acquired UTIs presenting to this hospital.

\section{Materials and Methods}

The study was carried out during a four month period from January $01^{\text {st }}$ to April $30^{\text {th }}$ in 2009 . Significant isolates from urine samples of adult patients received for routine culture were included in the study. Sample processing was done according to standard microbiological culture methods and species identification carried out using standard biochemical tests. Isolates were considered significant if there was a pure growth of $\geq 10^{5} \mathrm{cfu} / \mathrm{ml}$ or a pure growth of $10^{4}$ $10^{5} \mathrm{cfu} / \mathrm{ml}$ in a symptomatic patient. Presence or absence of pus cells was not considered for this decision. Antibiotic susceptibility testing (ABST) was performed according to the disk diffusion test recommendations of the Clinical Laboratory Standards Institute ${ }^{6}$ for ampicillin, nalidixic acid, nitrofurantoin, norfloxacin, co-trimoxazole, gentamicin, amoxicillin-clavulanate, cefuroxime, ciprofloxacin, amikacin, netilmicin, imipenem, cefotaxime and ceftazidime. Screening for ESBL production was by using cefotaxime and ceftazidime disks and confirmation by the combination disk method using cefotaxime and ceftazidime with and without clavulanic acid. ${ }^{6}$ Quality control of antibiotic disks and media was performed according to the CLSI guidelines $(2008)^{6}$ and CLSI recommended reference strains were used as positive and negative controls for ESBL detection.

Demographic details of the patients and any associated risk factors were obtained by referring to request forms, bed head tickets and by directly interviewing the patients. 
Community acquired urinary tract infections were defined as follows; in the out-patient department (OPD), patients who had no history of hospital admissions during the past three months and in hospitalized patients, patients whose urine cultures were positive within 48 hours of admission and who had no history of hospital admissions during the past three months.

\section{Results}

A total of 2303 urine samples were received during the study period and the number of significant isolates was $626(27.18 \%)$. Of the $286(46 \%)$ isolates included in the study, 228 $(80 \%)$ were E. coli and $58(20 \%)$ were Klebsiella species.

Most of the E. coli and Klebsiella isolates included in the study (211/286) were from general medical and surgical wards. There were 56 isolates from the outpatient department (OPD). The number of isolates of these species from intensive care units (ICU) and the genitourinary (GU) unit was small (11 and 8 respectively). A majority of the isolates included in the study were from female patients $(n=215,75 \%)$ and community acquired $(n=181,63 \%)$ infections.

The ESBL screening test was positive in 100 isolates of which 94 were positive by the confirmatory test. Half (50\%) of the Klebsiella isolates were ESBL producers and $29 \%$ of E. coli produced ESBL. 71 of 105 isolates (68\%) causing hospital acquired infections and 23 of 181 isolates (13\%) causing community acquired infections were ESBL producers.

ESBL producers were isolated from 70 of 211(33\%) patients admitted to the general medical and surgical wards. Ten of the 11 isolates from the ICU were ESBL producers. In the GU unit, 6 of 8 isolates produced ESBL. UTI in 8 of $56(14 \%)$ patients presenting to the outpatient department was caused by ESBL producers. The ESBL rate among out-patients was significantly less compared to ICU $(\mathrm{P}<0.001)$, GU unit $(\mathrm{P}<0.05)$ and general wards $(\mathrm{P}<0.05)$.

The mean age of patients with an ESBL positive isolate was 54 years vs. 45 in ESBL nonproducers. The mean number of days in the hospital prior to the positive urine culture for patients with an ESBL positive isolate was 4.1 days and that for patients with an ESBL negative isolate was 1.7 days.

Presence of urinary catheters, diabetes mellitus, previous antibiotic use, urinary tract abnormalities (renal calculi, prostate enlargement, hydronephrosis, hydroureter and polycystic kidney) and recurrent urinary tract infections had statistically significant associations with acquiring ESBL producing organisms $(\mathrm{P}<0.001)$ (Table 1).

Overall, resistance to commonly used oral antibiotics was high among both ESBL positive as well as ESBL negative coliforms (ampicillin 100\% and 77\%; nalidixic acid 98\%, and 45\%; norfloxacin $97 \%$ and 29\%; co-trimoxazole $79 \%$ and $23 \%$ ). ESBL producing coliforms had resistance rates of $19 \%, 72 \%, 61 \% .94 \%, 21 \%$ and $38 \%$ for nitrofurantoin, gentamicin, coamoxyclav, ciprofloxacin, amikacin and netilmicin respectively compared to isolates that were negative for ESBL with resistance rates of $1 \%, 2 \%, 2 \%, 8 \%, 0.5 \%$ and $1 \%$ respectively. One 
isolate (from an ICU patient) was resistant to imipenem. Carbapenemase detection was not done for this isolate.

Table 1. Risk factor associations

\begin{tabular}{|c|c|c|c|c|}
\hline Risk factor & & $\begin{array}{l}\text { No. of ESBL (+) } \\
\text { isclates }\end{array}$ & $\begin{array}{l}\text { No. of ESBL (-) } \\
\text { isclates }\end{array}$ & Pvalue \\
\hline \multirow[t]{2}{*}{ Urinary catheter } & $\gamma_{e s}$ & 27 & 8 & \multirow[t]{2}{*}{$<0.00 \mid$} \\
\hline & $\mathrm{No}$ & 62 & 178 & \\
\hline \multirow[t]{2}{*}{ Previous use of antibiotics } & Yes & 64 & 19 & \multirow[t]{2}{*}{$<0.001$} \\
\hline & $\mathrm{No}$ & 25 & 167 & \\
\hline \multirow[t]{2}{*}{ Urinary tract abnormalities } & Yes & 24 & 8 & \multirow[t]{2}{*}{$<0.00 \mid$} \\
\hline & $\mathrm{No}$ & 65 & 178 & \\
\hline \multirow[t]{2}{*}{ Recurrent UTIs } & $\gamma_{\text {es }}$ & 18 & 6 & \multirow[t]{2}{*}{$<0.00 \mid$} \\
\hline & $\mathrm{No}$ & 71 & 180 & \\
\hline \multirow[t]{2}{*}{ Diabetes mellitus } & Yes & 36 & 20 & \multirow[t]{2}{*}{$<0.00 \mid$} \\
\hline & $\mathrm{No}$ & 53 & 166 & \\
\hline
\end{tabular}

Overall, resistance to commonly used oral antibiotics was high among both ESBL positive as well as ESBL negative coliforms (ampicillin 100\% and 77\%; nalidixic acid 98\%, and 45\%; norfloxacin $97 \%$ and 29\%; co-trimoxazole $79 \%$ and $23 \%$ ). ESBL producing coliforms had resistance rates of $19 \%, 72 \%, 61 \% .94 \%, 21 \%$ and $38 \%$ for nitrofurantoin, gentamicin, coamoxyclav, ciprofloxacin, amikacin and netilmicin respectively compared to isolates that were negative for ESBL with resistance rates of $1 \%, 2 \%, 2 \%, 8 \%, 0.5 \%$ and $1 \%$ respectively. One isolate (from an ICU patient) was resistant to imipenem. Carbapenemase detection was not done for this isolate.

Nitrofurantoin was an effective oral agent for infections with coliforms in the general medical and surgical wards (5\% resistance) and OPD (3\% resistance) but there was relatively high resistance in the ICU and the GU unit (36\% and 25\% respectively). Co-amoxyclav was another oral agent which retained usefulness for coliforms in the general wards and OPD setting (resistance rates $9 \%$ and $12.5 \%$ respectively). Ciprofloxacin and norfloxacin had high resistance rates in all units.

There were 181 community acquired isolates among which $84 \%(\mathrm{n}=152)$ were E. coli. The ESBL positivity rate was $13 \%(n=23)$. Eleven percent of $E$. coli isolates $(n=16)$ and $24 \%(n=7)$ of Klebsiella isolates produced ESBL. Community acquired coliform isolates showed a 79\% resistance rate to ampicillin while resistance to nalidixic acid, norfloxacin, co-trimoxazole, coamoxyclav and cefuroxime was 50\%, 34\%, 26\%, 8\% and 15\% respectively. All isolates were sensitive to imipenem and only two isolates each were resistant to amikacin and nitrofurantoin. Notably, resistance to ciprofloxacin was high (17\%). 
Table 2. Antibiotic resistance rates of E coli and Klebsiella spp according to units

\begin{tabular}{|c|c|c|c|c|c|c|c|c|}
\hline & \multirow{2}{*}{\multicolumn{2}{|c|}{$\begin{array}{l}\text { ICU } \\
(\mathrm{n}=11)\end{array}$}} & \multirow{2}{*}{\multicolumn{2}{|c|}{$\begin{array}{l}\text { [U } \\
(n=8)\end{array}$}} & \multirow{2}{*}{\multicolumn{2}{|c|}{$\begin{array}{c}\mathrm{OPD} \\
(\mathrm{n}=56)\end{array}$}} & \multirow{2}{*}{\multicolumn{2}{|c|}{$\begin{array}{l}\text { General medical and surgical } \\
\text { wards ( } \mathrm{n}=211)\end{array}$}} \\
\hline & & & & & & & & \\
\hline & №. resistant & $\begin{array}{c}\text { Resistance } \\
\text { rate } \% \\
\end{array}$ & No. resistant & $\begin{array}{c}\text { Resistance } \\
\text { rate } \% \\
\end{array}$ & №. resistant & $\begin{array}{c}\text { Resistance } \\
\text { rate \% }\end{array}$ & №. resistant & $\begin{array}{c}\text { Resistance } \\
\text { rate \% }\end{array}$ \\
\hline Ampicillin & $\|$ & 100 & 8 & 100 & 41 & 73 & 182 & 86 \\
\hline Nalidixic acid & $\|$ & 100 & 8 & 100 & 23 & 41 & 210 & 99 \\
\hline Norflaxacin & 10 & 91 & 8 & 100 & 16 & 28 & 112 & 53 \\
\hline Cefuraxime & 10 & 9I & 5 & 62.5 & 9 & 16 & 74 & 35 \\
\hline Ca-trimoxazole & 10 & II & 7 & 87.5 & 15 & 26.7 & 86 & 40.7 \\
\hline Co-amaxyclav & 9 & 82 & 3 & 37.5 & 7 & 12.5 & 42 & 19 \\
\hline Ciprofloxacin & 9 & 82 & 7 & 87.5 & 12 & 21 & 75 & 35.5 \\
\hline Cefotaxime & 9 & 82 & 2 & 25 & 8 & 14.2 & 63 & 29.8 \\
\hline Gentamicin & 8 & 73 & 4 & 50 & 9 & 16 & 51 & 24 \\
\hline Ceftazidime & 8 & 73 & 3 & 37.5 & 7 & 12.5 & 60 & 28.4 \\
\hline Nitrufurantain & 4 & 36 & 2 & 25 & 2 & 3 & 12 & 05 \\
\hline Netilmicin & 4 & 36 & 1 & 12.5 & 8 & 14 & 26 & 12 \\
\hline Amikacin & 2 & 18 & 1 & 12.5 & 3 & 05 & 15 & 07 \\
\hline Imipenem & 1 & 09 & 0 & 0 & 0 & 0 & 0 & 0 \\
\hline
\end{tabular}

ESBL positive community acquired isolates exhibited a high degree of resistance to multiple antibiotics compared to ESBL negative isolates. The percentages of ESBL positive isolates with resistance to cefuroxime, co-amoxyclav, gentamicin and ciprofloxacin were $91 \%, 52 \%, 48 \%$ and $96 \%$ while the resistance rates of ESBL negatives were $4 \%, 0.63 \%, 2 \%$ and $5 \%$ respectively.

\section{Discussion}

E. coli and Klebsiella sp. accounted for only $46 \%$ of significant isolates from UTI in this setting and were mainly isolated from patients in the out-patient department and the general medical and surgical wards. UTI in the ICU and the GU unit were more often due to non-coliform bacteria. As has been previously described in many studies, UTI due to E. coli and Klebsiella spp. was mainly community acquired and was more common in women than in men.

A high rate of ESBL production was seen in E. coli and Klebsiella spp causing hospital acquired UTI. Coliforms isolated from UTI were very likely to be ESBL producers if they were isolated in the ICU or GU setting or from patients in general medical and surgical wards who presented with hospital acquired infections. However isolates from community acquired UTI presenting to the OPD or admitted to the general wards were significantly less likely to be due to ESBL producers. The risk factors identified in this study as having a statistically significant association with UTI caused by ESBL producing coliforms such as duration of admission prior to positive culture, presence of urinary catheters, diabetes mellitus, previous antibiotic use, urinary tract 
abnormalities, recurrent urinary tract infections and increasing age have been previously well documented. $^{2,4,7}$

Resistance rates to commonly used oral antibiotics were high in both groups. When compared to the ESBL negative isolates, the ESBL positive isolates were highly resistant to multiple antibiotics. Such co-resistance to multiple drug classes is well documented in ESBL producers. ${ }^{2,4,8}$

With regard to the resistance patterns seen in the various units, nitrofurantoin and co-amoxyclav retained acceptable sensitivity in the general medical and surgical wards and OPD setting. Although coliforms were not a common cause of UTI in the ICU and the GU unit, an alarmingly high level of drug resistance in such isolates was noted. In these units, patients have complicated illnesses, undergone various interventions and invasive procedures and high antibiotic consumption which predispose to increased ESBL prevalence and high drug resistance rates.

Ciprofloxacin and norfloxacin, which are commonly used in the treatment of UTI in our hospital, especially in the GU unit, showed unacceptably high resistance in all units. This fact has been observed by other investigators and raises the question of the appropriateness of fluoroquinolones for empirical therapy. ${ }^{9}$

The proportion of ESBL producers in community acquired E. coli and Klebsiella spp. causing UTI (13\%) was relatively low when compared to most neighboring countries but higher than that in the European countries. ${ }^{10,11,12}$ Community acquired isolates showed a favorable sensitivity pattern other than for those isolates which were ESBL producers.

\section{Conclusion}

This study demonstrates the importance of regular review of empirical antibiotic therapy for UTI in view of the evolving resistance of uropathogens to commonly used agents. Choice of antibiotics based on the hospital unit, source of UTI, presence of risk factors and current sensitivity patterns is recommended. Since community acquired UTI is seen in a variety of settings including general practice, continuing surveillance in different settings is needed to determine the burden of ESBL producing organisms. This would assist in developing strategies for curtailing emergence and spread of ESBL producers as well as in decision making for empirical antibiotic treatment in different settings.

Conflicts of interest - None exist.

\section{References}

1. Ronald, A. The etiology of urinary tract infection: traditional and emerging pathogens. Am J Med. 2002; Suppl 1A:113 :14S-19S. No doi 
2. Bradford, P.A. Extended-spectrum B-lactamases in the 21st century: characterization, epidemiology, and detection of this important resistance threat. Clinical Microbiology Review. 2001; 14: 933-51 doi: 10.1128/CMR.14.4.933-951.2001

3. Arpin, C., Quentin, C., Grobost, F., Cambau, E., Robert, J., Dubois, V., Coulange, L., Andre, C. and on behalf of the Scientific Committee of ONERBA Nationwide survey of extendedspectrum \{beta\}-lactamase-producing Enterobacteriaceae in the French community setting .J. Antimicrob. Chemother. 2009; 63 (6): 1205 - 1214 doi: 10.1093/jac/dkp108

4. Pitout, J.D.D., Laupland, K,B. Extended-spectrum $\beta$-lactamase producing enterobacteriaceae: an emerging public health concern. Lancet Infect Dis. 2008; 8: 159-166. doi:10.1016/S1473-3099(08)70041-0

5. Hanson, N. D., Moland, E. S., Hong, S. G., Propst, K., Novak, D. J. and Cavalieri, S. J. Surveillance of community-based reservoirs reveals the presence of CTX-M, imported AmpC, and OXA-30 \{beta\}-Lactamases in urine isolates of Klebsiella pneumoniae and Escherichia coli in a U.S. community. Antimicrob. Agents Chemother. 2008; 52(10):38143816. doi:10.1128/AAC.00877-08

6. Clinical and Laboratory Standards Institute. Performance Standards for Antimicrobial Susceptibility Testing; Eighteenth Informational Supplement M100-S18. Clinical and Laboratory Standards Institute, Wayne, PA, USA, 2008.

7. Hart, C. A. Pocket Guide to Extended-Spectrum \{beta\}-Lactamases in Resistance - D. M. Livermore and D. L. Paterson. Current Medicine Group, London, UK, 2006. Oxford University Press. ISBN 1-85873-859-8.

8. Babypadmini, S. \& Appalaraju, B. Extended spectrum -lactamases in urinary isolates of Escherichia coli and Klebsiella pneumoniae - Prevalence and susceptibility pattern in a tertiary care hospital. Indian Journal of Medical Microbiology. 2004; 22: 172-174. No doi

9. Wijekoon, C.N., Dassanayake, K.M.M.P., Perera, W.P.M.H., Pathmeswaran, A. Antimicrobial susceptibility of urinary isolates and prescribing practices of empirical antimicrobials in patients with urinary tract infection in a tertiary care hospital. The bulletin of the Sri Lanka College of Microbiologists. 2009; 7:15. No doi

10. Gupta, V., Yadav, A., Joshi, R.M. Antibiotic resistance pattern in uropathogens. Indian J Med Microbiol._2002; 20 : 96-8. No doi

11. Colodner, R., Keness, Y., Chazan, B. et al. Antimicrobial susceptibility of communityacquired uropathogens in northern Israel. Int J Antimicrob Agents 2001; 18: 189-92. doi:10.1016/S0924-8579(01)00368-5

12. Rodriguez-Bano, J., Alcala, J. C., Cisneros, J. M., Grill, F., Oliver, A., Horcajada, J. P., Tortola, T., Mirelis, B., Navarro, G., Cuenca, M., Esteve, M., Pena, C., Llanos, A. C., Canton, R., Pascual, A. . Community infections caused by extended-spectrum \{beta\}lactamase-producing Escherichia coli. Arch Intern Med. 2008;168: 1897-1902. doi:10.1001/archinte.168.17.1897 